\title{
COMPREHENSIVE ANALYSIS OF MAGNETIC SUPERSTRUCTURES IN ANISOTROPIC SYSTEMS
}

\author{
N. Gonchar ${ }^{\dagger}$, W. Kozyrski ${ }^{\dagger \dagger}$, H. Hajduk \\ Department of Mathematical Modelling, N. Bogolubov Institute for Theoretical Physics, \\ Ukrainian National Academy of Sciences, 14 b Metrolohichna Str., Kyiv UA-252143, Ukraine \\ ${ }^{\dagger}$ E-mail: mgonchar@gluk.apc.org, ${ }^{\dagger \dagger}$ E-mail: kozyrski@gluk.apc.org
}

(Received August 12, 1996)

\begin{abstract}
New method is developed for analytical treatment of modulated superstructures in model magnetic systems near criticality. We found the conditions for paramagnet-(in)commensurate transition in the class of anisotropic Ising models with and without an external magnetic field and the critical value of the latter. We derived a new representation of specific free energy for commensurate and incommensurate configurations and found its hidden symmetry. The existence of the global solution is proved for the incommensurate configuration minimizing free energy. We found the condition for the global solution's termination. The existence of phase transition from the incommensurate phase into the ferromagnetic one is proved to depend on exchange integrals.
\end{abstract}

Key words: magnetic systems; modulated superstructures, hidden symmetry, paramagnet(in)commensurate transition, critical field.

PACS number(s):05.50.+q, 64.60.Cn

\section{INTRODUCTION}

The importance of a comprehensive theoretical treatment of modulated structures is well known [1]. The difficulties of studying the phase diagram for such systems are caused by the need to continue the free energy per lattice site from the commensurate configurations to incommensurate ones and constructing the extremum configurations. The interest to this problem does not diminish with time [2-7]. Here we deliver a new treatment of modulated structures in anisotropic Ising system near criticality. We derive new representation for free energy of both commensurate and incommensurate configurations and find its hidden symmetry (Sec.II). In Sec.III and IV we prove the existence of phase transition from the paramagnetic state to the modulated structures with and without external magnetic field. In Sec.IV we find the extreme magnetic field for which modulated structures still exist. In Sec.V we prove the existence of the global solution for the incommensurate configuration minimizing the free energy and derive the condition for this solution to terminate. In Sec.V the existence of the phase transition from the incommensurate phase into the ferromagnetic one is proved to depend on exchange integrals. This phase transition is of the first kind.

To be definite we illustrate the method on the ANNNI model [8] but one can apply it for models with an arbitrary number of interacting neighbors. The mean-field free energy for three-dimensional ANNNI model is

$$
\bar{F}_{h}=\sum_{i<j} J_{i j} m_{i} m_{j}+\frac{1}{2} T \sum_{i}\left(\left(1+m_{i}\right) \ln \left(1+m_{i}\right)\right.
$$

$$
\left.+\left(1-m_{i}\right) \ln \left(1-m_{i}\right)\right)+h \sum_{i} m_{i},
$$

where $m_{i}$ is an average magnetic moment at $i$-th site and takes values on the segment $[-1,1]$. In the planes normal to the $x$-axis the nearest neighbors only interact ferromagnetically $\left(J_{0}<0\right)$. Along the axis $x$ the nextnearest neighbors interact too, moreover the interactions of the nearest neighbors $\left(J_{1}\right)$ and the next-nearest ones $\left(J_{2}\right)$ can be either ferromagnetic or antiferromagnetic. Just the relations between them are essential. Expression (1.1) is formal because specific free energy only has the physical sense. Let us derive the explicit expression for it.

Denote by $\Lambda_{0}$ the parallelepiped from $\mathcal{R}^{3}$ of sizes $a N_{1}, a N_{2}$ and $a N_{3}$, where $a$ is lattice spacing, $N_{1} \times N_{2} \times$ $N_{3}$ is the number of sites in $\Lambda_{0}$. We consider the set of commensurate configurations on the integer-valued lattice $\mathcal{Z}^{3}$ with the periods $N_{1}, N_{2}$ and $N_{3}$. Denote by $\left\{\Lambda_{k}\right\}$ the sequence of volumes from $\mathcal{R}^{3}$ with $N_{k}$ sites from $\mathcal{Z}^{3}$ and cover the volume $\Lambda_{k}$ by parallelepipeds congruent to $\Lambda_{0}$ with their number inside $\Lambda_{k}$ being $n_{1}^{k}$ and the one completely covering $\Lambda_{k}$ being $n_{2}^{k}$. If the sequence $\Lambda_{k}$ tends to $\mathcal{R}^{3}$ and $\Lambda_{k} \subseteq \Lambda_{k+1}, \bigcup_{k=1}^{\infty} \Lambda_{k}=\mathcal{R}^{3}$, then

$$
\lim _{k \rightarrow \infty} \frac{\left(n_{2}^{k}-n_{1}^{k}\right) N_{1} N_{2} N_{3}}{\left|\Lambda_{k}\right|}=0
$$

where $\left|\Lambda_{k}\right|=N_{k}$ is the number of sites in $\Lambda_{k}$.

The specific free energy for commensurate configurations in $\Lambda$ of the periods $N_{1}, N_{2}, N_{3}$ with boundary condition over $\Lambda$ takes the form 


$$
\begin{aligned}
\frac{\bar{F}_{h k}}{\left|\Lambda_{k}\right|} & =\frac{1}{2\left|\Lambda_{k}\right|} \sum_{j=1}^{n_{1}^{k}} \sum_{i \in \Lambda_{o j}}\left(m _ { i } \left(J_{1}\left(m_{i+1}^{o x}+m_{i-1}^{o x}\right)\right.\right. \\
& \left.+J_{2}\left(m_{i+2}^{o x}+m_{i-2}^{o x}\right)+J_{0}\left(m_{i+1}^{o y}+m_{i-1}^{o y}\right)+J_{0}\left(m_{i+1}^{o z}+m_{i-1}^{o z}\right)\right) \\
& \left.+2 h m_{i}+T\left(\left(1+m_{i}\right) \ln \left(1+m_{i}\right)+\left(1-m_{i}\right) \ln \left(1-m_{i}\right)\right)\right)+\frac{\bar{F}_{1}^{k}}{\left|\Lambda_{k}\right|}
\end{aligned}
$$

where the contribution of covering volumes $n_{2}^{k}-n_{1}^{k}$ accounting for the boundary conditions is denoted by $\bar{F}_{1}^{k}$. $m_{i+1}^{o x}, m_{i-1}^{o x}, m_{i+1}^{o y}, m_{i-1}^{o y}$ and so on are the average magnetic moments at sites $i+1, i-1$ along the corresponding axes.

The thermodynamical limit of specific free energy for commensurate configurations is equal to

$$
\begin{aligned}
F_{h} & =\lim _{k \rightarrow \infty} \frac{\bar{F}_{h k}}{\left|\Lambda_{k}\right|}=\lim _{k \rightarrow \infty} \frac{n_{1}^{k}}{2\left|\Lambda_{k}\right|} \sum_{i \in \Lambda_{o}}\left(m _ { i } \left(J_{1}\left(m_{i+1}^{o x}+m_{i-1}^{o x}\right)\right.\right. \\
& \left.+J_{2}\left(m_{i+2}^{o x}+m_{i-2}^{o x}\right)+J_{0}\left(m_{i+1}^{o y}+m_{i-1}^{o y}\right)+J_{0}\left(m_{i+1}^{o z}+m_{i-1}^{o z}\right)\right)+2 h m_{i} \\
& \left.+T\left(\left(1+m_{i}\right) \ln \left(1+m_{i}\right)+\left(1-m_{i}\right) \ln \left(1-m_{i}\right)\right)\right)+\lim _{k \rightarrow \infty} \frac{\bar{F}_{1}^{k}}{\left|\Lambda_{k}\right|}
\end{aligned}
$$

or $[9]$

$$
\begin{aligned}
F_{h} & =\frac{1}{2 N_{1} N_{2} N_{3}} \sum_{i \in \Lambda_{o}}\left(m _ { i } \left(J_{1}\left(m_{i+1}^{o x}+m_{i-1}^{o x}\right)\right.\right. \\
& \left.+J_{2}\left(m_{i+2}^{o x}+m_{i-2}^{o x}\right)+J_{0}\left(m_{i+1}^{o y}+m_{i-1}^{o y}\right)+J_{0}\left(m_{i+1}^{o z}+m_{i-1}^{o z}\right)\right) \\
& \left.+2 h m_{i}+T\left(\left(1+m_{i}\right) \ln \left(1+m_{i}\right)+\left(1-m_{i}\right) \ln \left(1-m_{i}\right)\right)\right) .
\end{aligned}
$$

Actually, the quasi-one-dimensional case is of interest, i.e., the configurations with the fixed average magnetic moment at the sites of planes parallel to the plane $Y O Z$. The latter is valid for $J_{0}<0$ and a sufficiently low temperature $T$. In that case specific free energy with $m_{i+1}^{o y}=m_{i-1}^{o y}=m_{i+1}^{o z}=m_{i-1}^{o z}=m_{i}$ takes the form of

$$
\begin{aligned}
F_{h} & =\frac{1}{2 N_{1}} \sum_{i \in \Lambda_{\circ}^{\prime}}\left(m_{i}\left(J_{1}\left(m_{i+1}+m_{i-1}\right)+J_{2}\left(m_{i+2}+m_{i-2}\right)+4 J_{0} m_{i}\right)\right. \\
& \left.+2 h m_{i}+T\left(\left(1+m_{i}\right) \ln \left(1+m_{i}\right)+\left(1-m_{i}\right) \ln \left(1-m_{i}\right)\right)\right)
\end{aligned}
$$

where $\Lambda_{o}^{\prime}$ is a piece of line with $N_{1}$ sites.

\section{UNDERLYING SYMMETRY OF FREE ENERGY}

Here we find the hidden one-parameter symmetry group of the free energy per lattice site in the space of commensurate and incommensurate configurations. At first we construct the new representation for specific free energy (1.3) of commensurate configurations and extend it onto the set of incommensurate ones. To do this we introduce the set of vectors $X=\left\{x_{i}\right\}_{i=-\infty}^{+\infty}$, where $i \in \mathcal{Z}_{1}$ and $x_{i}$ takes values in the set of real numbers, and the scalar product 


$$
\left\langle x_{1}, x_{2}\right\rangle=\lim _{\varepsilon \rightarrow 0} \frac{\sum_{i=-\infty}^{+\infty} e^{-\varepsilon|i|} x_{i}^{(1)} x_{i}^{(2)}}{\sum_{i=-\infty}^{+\infty} e^{-\varepsilon|i|}}
$$

with $x \in X$, if $\|x\|^{2}=\langle x, x\rangle<+\infty$. The vector is periodic of the period $N_{1}$ if $x_{i+N_{1}}=x_{i}$ for $\forall i \in \mathcal{Z}_{1}$. Let $N_{1}$ be such an integer that $N=\left[N_{1} / 2\right]$, where $[c]$ is the integral part of $c$, and $q=m / N_{1}$ is irreducible quotient. Next we introduce

$$
\begin{gathered}
\epsilon=\left\{a_{i}\right\}_{i=-\infty}^{+\infty}, \quad a_{i}=1 \text { for } \forall i \\
e_{1}(q)=\{\cos i 2 \pi q\}_{i=-\infty}^{+\infty}, \quad e_{2}(q)=\{\sin i 2 \pi q\}_{i=-\infty}^{+\infty} .
\end{gathered}
$$

Vectors

$$
\left\{\epsilon, e_{1}(q), \cdots, e_{1}(N q), e_{2}(q), \cdots, e_{2}(N q)\right\}
$$

belong to the set $X$, form the orthogonal system and are periodic of the period $N_{1}$. Any $N_{1}$-periodic configuration $x$ can be expressed as

$$
x=\sum_{l=1}^{N}\left(a_{l} e_{1}(l q)+b_{l} e_{2}(l q)\right)+a_{0} \epsilon .
$$

Lemma 1. For configuration (2.1) the formula

$$
\langle x, \epsilon\rangle=\frac{1}{N_{1}} \sum_{i=1}^{N_{1}} x_{i}
$$

holds, where $x_{i}$ is $i$-th component of the vector $x$.

For the proof see [10].

If $g(x)$ is some continuous function of $x \in[a, b]$, then

$$
g(x)=\lim _{n \rightarrow \infty} P_{n}(x)
$$

where $P_{n}(x)$ is the sequence of polynomials approximating $g(x)$ on $[a, b]$ uniformly. We consider the function

$$
g(x)=(1+x) \ln (1+x)+(1-x) \ln (1-x)
$$

and its derivatives. If the configuration $x=\left\{x_{i}\right\}_{i=-\infty}^{+\infty}$ is such that

$$
\left|x_{i}\right|<1 \quad \forall i=\overline{-\infty,+\infty}
$$

then $g(x)$ is defined correctly.

Using representation (4.1) for any $N_{1}$-periodic configuration we calculate the averages

$$
I_{s, m}=\left\langle\prod_{k=1}^{s} e_{1}\left(i_{k} q\right) \prod_{l=1}^{m} e_{2}\left(j_{l} q\right), \epsilon\right\rangle
$$

for any power $n$ of this configuration. For $q=m / N_{1}$ the averages $I_{s, m}$ are expressed as [10]

$$
I_{s, m}=\left\{\begin{array}{cc}
0, \quad m=2 M+1, \quad M=0,1,2, \cdots, & \\
& \sum \delta_{t_{1}+\cdots+t_{s}+\tau_{1}+\cdots+\tau_{m},|r| N_{1}}(-1)^{k} \\
(-1)^{M} 2^{-s-2 m} \sum_{r=0, \pm 1, \cdots, \pm\left[\frac{l}{N_{1}}\right]} t_{k^{\prime}}= \pm i_{k^{\prime}} & \\
\tau_{l}= \pm j_{l} & m=2 M
\end{array}\right.
$$

$k$ is the number of negative $\tau_{l}$. For irrational $q$ the averages $I_{s, m}$ are expressed by the formula

$$
I_{s, m}=\left\{\begin{aligned}
0, \quad m=2 M+1, \quad M=0,1,2, \cdots \\
(-1)^{M} 2^{-s-2 m} \sum \delta_{t_{1}+\cdots+t_{s}+\tau_{1}+\cdots+\tau_{m}, 0}(-1)^{k}, m=2 M \\
t_{k^{\prime}}= \pm i_{k^{\prime}} \\
\tau_{l}= \pm j_{l}
\end{aligned}\right.
$$

Due to Lemma 1 the specific free energy (1.3) has the form $F_{h}=\langle\epsilon, f(m)\rangle / 2$, where $f(m)=\left\{f\left(m_{i}\right)\right\}_{i=-\infty}^{+\infty}$ with

$$
\begin{aligned}
f\left(m_{i}\right) & =m_{i}\left(J_{1}\left(m_{i+1}+m_{i-1}\right)+J_{2}\left(m_{i+2}+m_{i-2}\right)+4 J_{0} m_{i}+2 h\right) \\
& +T\left(\left(1+m_{i}\right) \ln \left(1+m_{i}\right)+\left(1-m_{i}\right) \ln \left(1-m_{i}\right)\right) .
\end{aligned}
$$


For

$$
m=\sum_{l=1}^{N}\left(a_{l} e_{1}(l q)+b_{l} e_{2}(l q)\right)+a_{0} \epsilon
$$

we have

$$
\begin{aligned}
F_{h} & =-\frac{1}{2}\langle m, A m\rangle+h\langle\epsilon, m\rangle+\frac{1}{2} T\langle\epsilon, g(m)\rangle=-\frac{1}{2}\left(\frac{1}{2} \sum_{l=1}^{N}\left(\left(1+\delta_{l,\left[\frac{N_{1}+1}{2}\right]}\right) a_{l}^{2}+\left(1-\delta_{l,\left[\frac{N_{1}+1}{2}\right]}\right) b_{l}^{2}\right) \Phi(l q)\right. \\
& \left.+a_{0}^{2} \Phi(0)\right)+h a_{0}+\frac{1}{2} T\left\langle\epsilon, g\left(\sum_{l=1}^{N}\left(a_{l} e_{1}(l q)+b_{l} e_{2}(l q)\right)+a_{0} \epsilon\right)\right\rangle
\end{aligned}
$$

where $A$ is the operator defined as

$$
(A m)_{i}=-\left(J_{1}\left(m_{i+1}+m_{i-1}\right)+J_{2}\left(m_{i+2}+m_{i-2}\right)+4 J_{0} m_{i}\right)
$$

and the function $g$ is

$$
g(m)=(1+m) \ln (1+m)+(1-m) \ln (1-m)=\lim _{n \rightarrow \infty} 2\left(\frac{m^{2}}{2}+\frac{m^{4}}{3 \cdot 4}+\cdots+\frac{m^{2 n}}{2 n \cdot(2 n-1)}\right)
$$

provided that

$$
\sum_{l=1}^{N}\left(\left|a_{l}\right|+\left|b_{l}\right|\right)+\left|a_{0}\right|<1
$$

For the ANNNI model

$$
\Phi(q)=-2\left(J_{1} \cos 2 \pi q+J_{2} \cos 4 \pi q+2 J_{0}\right)
$$

and for the case when all the neighbors interact

$$
\Phi(q)=-2\left(\sum_{i=1}^{+\infty} J_{i} \cos 2 \pi i q+2 J_{0}\right)
$$

The free energy (2.6) is defined on the set of commensurate configuratons. The following Lemma defines specific free energy on the set of incommensurate configurations.

Lemma 2. The thermodynamical limit of the free energy exists for irrational $q$ on the set of incommensurate configurations

$$
m=\sum_{l=1}^{+\infty}\left(a_{l} e_{1}(l q)+b_{l} e_{2}(l q)\right)+a_{0} \epsilon
$$

provided that

$$
\sum_{l=1}^{+\infty}\left(\left|a_{l}\right|+\left|b_{l}\right|\right)+\left|a_{0}\right|<1
$$

For the proof see [10].

As a result free energy of configuration (2.1) with irrational $q$ and arbitrary $N$ is

$$
F_{h}=-\frac{1}{2}\left(\frac{1}{2} \sum_{l=1}^{N}\left(a_{l}^{2}+b_{l}^{2}\right) \Phi(l q)+a_{0}^{2} \Phi(0)\right)+h a_{0}+\frac{1}{2} T\left\langle\epsilon, g\left(\sum_{l=1}^{N}\left(a_{l} e_{1}(l q)+b_{l} e_{2}(l q)\right)+a_{0} \epsilon\right)\right\rangle
$$


Let us find the symmetry group of free energy (2.6) for commensurate configurations of the period $N_{1}$. To do this we apply the previous representation of free energy (1.3). Due to $N_{1}$-periodicity of $m=\left\{m_{i}\right\}_{i=-\infty}^{+\infty}$

$$
\begin{aligned}
F_{h} & =\frac{1}{2 N_{1}} \sum_{i=1}^{N_{1}}\left(m_{i}\left(J_{1}\left(m_{i+1}+m_{i-1}\right)+J_{2}\left(m_{i+2}+m_{i-2}\right)+4 J_{0} m_{i}\right)\right. \\
& \left.+2 h m_{i}+T g\left(m_{i}\right)\right)=\frac{1}{2 N_{1}} \sum_{i=1}^{N_{1}}\left(m _ { i + s } \left(J_{1}\left(m_{i+s+1}+m_{i+s-1}\right)\right.\right. \\
& \left.\left.+J_{2}\left(m_{i+s+2}+m_{i+s-2}\right)+4 J_{0} m_{i+s}\right)+2 h m_{i+s}+T g\left(m_{i+s}\right)\right)=F_{h}^{s} .
\end{aligned}
$$

Exploiting this invariance in the new representation we convert expression (2.8) into

$$
\begin{aligned}
F_{h}^{s} & =-\frac{1}{2}\left\langle\sum_{l=1}^{N}\left(a_{l} e_{1}^{s}(l q)+b_{l} e_{2}^{s}(l q)\right)+a_{0} \epsilon, \sum_{l=1}^{N}\left(a_{l} e_{1}^{s}(l q)+b_{l} e_{2}^{s}(l q)\right) \Phi(l q)+a_{0} \Phi(0) \epsilon\right\rangle \\
& +h\left\langle\epsilon, \sum_{l=1}^{N}\left(a_{l} e_{1}^{s}(l q)+b_{l} e_{2}^{s}(l q)\right)+a_{0} \epsilon\right\rangle+\frac{1}{2} T\left\langle\epsilon, g\left(\sum_{l=1}^{N}\left(a_{l} e_{1}^{s}(l q)+b_{l} e_{2}^{s}(l q)\right)+a_{0} \epsilon\right)\right\rangle .
\end{aligned}
$$

Using explicitly

$$
\begin{aligned}
& e_{1}^{s}(l q)=e_{1}(l q) \cos 2 \pi l s q-e_{2}(l q) \sin 2 \pi l s q \\
& e_{2}^{s}(l q)=e_{1}(l q) \sin 2 \pi l s q+e_{2}(l q) \cos 2 \pi l s q
\end{aligned}
$$

in (2.9) we have

$$
\begin{aligned}
F_{h} & =F_{h}^{s}=-\frac{1}{2}\left\langle\sum_{l=1}^{N}\left(a_{l}^{\prime} e_{1}^{s}(l q)+b_{l}^{\prime} e_{2}^{s}(l q)\right)+a_{0} \epsilon, \sum_{l=1}^{N}\left(a_{l}^{\prime} e_{1}^{s}(l q)+b_{l}^{\prime} e_{2}^{s}(l q)\right) \Phi(l q)+a_{0} \Phi(0) \epsilon\right\rangle \\
& +h\left\langle\epsilon, \sum_{l=1}^{N}\left(a_{l}^{\prime} e_{1}^{s}(l q)+b_{l}^{\prime} e_{2}^{s}(l q)\right)+a_{0} \epsilon\right\rangle+\frac{1}{2} T\left\langle\epsilon, g\left(\sum_{l=1}^{N}\left(a_{l}^{\prime} e_{1}^{s}(l q)+b_{l}^{\prime} e_{2}^{s}(l q)\right)+a_{0} \epsilon\right)\right\rangle,
\end{aligned}
$$

where

$$
\begin{aligned}
& a_{l}^{\prime}=a_{l} \cos 2 \pi l s q+b_{l} \sin 2 \pi l s q, \quad b_{l}^{\prime}=-a_{l} \sin 2 \pi l s q+b_{l} \cos 2 \pi l s q, \\
& q=m / N_{1}, \quad s=0,1, \cdots, N_{1} .
\end{aligned}
$$

So for rational $q$ free energy (2.6) is invariant under discrete transformation group (2.11). Here we describe the polynomial invariants of discrete rotation group. Consider averages

$$
\frac{1}{N_{1}} \sum_{s=1}^{N_{1}}\left(a_{1} \cos 2 \pi s q+b_{1} \sin 2 \pi s q\right)^{l}=P_{l}\left(a_{1}, b_{1}\right)
$$

$P_{l}\left(a_{1}, b_{1}\right)$ is homogeneous polynomial invariant. Really

$$
P_{l}\left(a_{1}^{\prime}, b_{1}^{\prime}\right)=\frac{1}{N_{1}} \sum_{s=1}^{N_{1}}\left(a_{1}^{\prime} \cos 2 \pi s q+b_{1}^{\prime} \sin 2 \pi s q\right)^{l}=\frac{1}{N_{1}} \sum_{s=1}^{N_{1}}\left(a_{1} \cos 2 \pi\left(s_{1}+s\right) q+b_{1} \sin 2 \pi\left(s_{1}+s\right) q\right)^{l}
$$




$$
=\frac{1}{N_{1}} \sum_{s=1}^{N_{1}}\left(a_{1} \cos 2 \pi s q+b_{1} \sin 2 \pi s q\right)^{l}=P_{l}\left(a_{1}, b_{1}\right) .
$$

After calculating the average $P_{l}\left(a_{1}, b_{1}\right)$ we have [11]

$$
\begin{aligned}
P_{l}\left(a_{1}, b_{1}\right) & =\frac{1}{N_{1}} \sum_{s=1}^{N_{1}}\left(a_{1}^{\prime} \cos 2 \pi s q+b_{1}^{\prime} \sin 2 \pi s q\right)^{l} \\
& =\frac{1}{2^{l}} \sum_{|r|=0}^{\left[\frac{l}{N_{1}}\right]}\left(a_{1}^{2}+b_{1}^{2}\right)^{\frac{l-|r| N_{1}}{2}} \sum_{k=0}^{l} C_{l}^{k} \delta_{|l-2 k|,|r| N_{1}} \sum_{m=0}^{\left[\frac{|r| N_{1}}{2}\right]}(-1)^{m} C_{|r| N_{1}}^{2 m} a_{1}^{|r| N_{1}-2 m} b_{1}^{2 m} .
\end{aligned}
$$

Now we find the symmetry of free energy (2.7) for irrational $q$. At first we derive new representation for the free energy. The polynomial coefficients

$$
I_{p}\left(a_{1}, b_{1}, \cdots, a_{N}, b_{N}, a_{0}\right)=\left\langle\epsilon,\left(\sum_{l=1}^{N}\left(a_{l} e_{1}(l q)+b_{l} e_{2}(l q)\right)+a_{0} \epsilon\right)^{p}\right\rangle
$$

are given by (2.5). The direct test gives

$$
\left\langle\prod_{k=1}^{s} e_{1}\left(i_{k} q\right) \prod_{l=1}^{m} e_{2}\left(j_{l} q\right)\right\rangle=\frac{1}{2 \pi} \int_{0}^{2 \pi} d \phi \prod_{k=1}^{s} \cos i_{k} \phi \prod_{l=1}^{m} \sin j_{l} \phi
$$

from where

$$
I_{p}\left(a_{1}, b_{1}, \cdots, a_{N}, b_{N}, a_{0}\right)=\frac{1}{2 \pi} \int_{0}^{2 \pi}\left(\sum_{l=1}^{N}\left(a_{l} \cos l \phi+b_{l} \sin l \phi\right)+a_{0}\right)^{p} d \phi
$$

$I_{p}$ is $2 \pi$-periodic function of $\phi$, therefore

$$
I_{p}\left(a_{1}^{\prime}, b_{1}^{\prime}, \cdots, a_{N}^{\prime}, b_{N}^{\prime}, a_{0}\right)=I_{p}\left(a_{1}, b_{1}, \cdots, a_{N}, b_{N}, a_{0}\right)
$$

where

$$
\begin{aligned}
& a_{l}^{\prime}=a_{l} \cos l \phi_{0}+b_{l} \sin l \phi_{0}, \quad b_{l}^{\prime}=-a_{l} \sin l \phi_{0}+b_{l} \cos l \phi_{0}, \\
& \phi_{0} \in[0,2 \pi] .
\end{aligned}
$$

Really

$$
\frac{1}{2 \pi} \int_{0}^{2 \pi}\left(\sum_{l=1}^{N}\left(a_{l} \cos l\left(\phi+\phi_{0}\right)+b_{l} \sin l\left(\phi+\phi_{0}\right)\right)+a_{0}\right)^{p} d \phi=\frac{1}{2 \pi} \int_{0}^{2 \pi}\left(\sum_{l=1}^{N}\left(a_{l} \cos l \phi+b_{l} \sin l \phi\right)+a_{0}\right)^{p} d \phi
$$

Therefore $I_{p}$ is invariant with respect to transformations (2.13) of group $G$ operating in a $2 N$-dimensional space. Due to the arbitrariness of $\phi$ and convergence of free energy series for small $a_{l}$ and $b_{l}$ the free energy (2.7) is invariant of group $G$. 


\section{NEAR CRITICAL MODULATED STRUCTURES $(H=0)$}

The existence problem for the extremum configurations is non-trivial. Here we study it for small deviations from the critical temperature. We prove the equivalence of the existence problem for a nonzero solution to equations for the configuration minimizing free energy and that for the corresponding branching equations. We derive the branching equations and prove the existence theorem for a nonzero solution. We investigate the model with an external magnetic field and without it. The necessary conditions of free energy minimum are equations

$$
\begin{aligned}
& -a_{l} \Phi(l q)\left(1+\delta_{l,\left[\frac{N_{1}+1}{2}\right]}\right)+T\left\langle\epsilon, g^{\prime}(x) e_{1}(l q)\right\rangle=0, \\
& -b_{l} \Phi(l q)\left(1-\delta_{l,\left[\frac{N_{1}+1}{2}\right]}\right)+T\left\langle\epsilon, g^{\prime}(x) e_{2}(l q)\right\rangle=0, \\
& -a_{0} \Phi(0)+h+T\left\langle\epsilon, g^{\prime}(x)\right\rangle / 2=0, \quad l=\overline{1, N},
\end{aligned}
$$

for rational $q$ and

$$
\begin{aligned}
& -a_{l} \Phi(l q)+T\left\langle\epsilon, g^{\prime}(x) e_{1}(l q)\right\rangle=0, \\
& -b_{l} \Phi(l q)+T\left\langle\epsilon, g^{\prime}(x) e_{2}(l q)\right\rangle=0, \\
& -a_{0} \Phi(0)+h+T\left\langle\epsilon, g^{\prime}(x)\right\rangle / 2=0 .
\end{aligned}
$$

for irrational $q$. Since

$$
\begin{aligned}
g^{\prime}(x) & =\ln \frac{1+x}{1-x} \\
& =2\left(x+\frac{x^{3}}{3}+\frac{x^{5}}{5}+\cdots+\frac{x^{2 n+1}}{2 n+1}+\cdots\right),
\end{aligned}
$$

one may denote

$$
g^{\prime}(x)=2 x+\varphi_{1}(x)
$$

where

$$
\begin{gathered}
\varphi_{1}(x)=2\left(\frac{x^{3}}{3}+\frac{x^{5}}{5}+\cdots+\frac{x^{2 n+1}}{2 n+1}+\cdots\right), \\
x \in(-1,1) .
\end{gathered}
$$

Lemma 3. For $x$ being a nontrivial configuration from Hilbert space of configurations, satisfying (3.1) or (3.2) for $h=0$, and $q$ satisfying inequality

$$
\Phi(q)>\Phi(l q), \quad l=0,2,3,4, \cdots, N-1, N, \quad \Phi(q)>0
$$

critical temperature $T_{0}$ does not exceed $\Phi(q)$, i.e. $T_{0} \leq$ $\Phi(q)$.
For the proof see [12].

Now consider the ANNNI model for $h=0$. In that case the critical temperature is equal to

$$
\begin{aligned}
T_{0} & =\sup _{0 \leq q \leq 1} \Phi(q) \\
& =\sup _{q}\left(-2 J_{1} \cos 2 \pi q-2 J_{2} \cos 4 \pi q-4 J_{0}\right)>0 .
\end{aligned}
$$

One can express $T_{0}$ in terms of $J_{1}, J_{2}, J_{0}$. From $\Phi^{\prime}(q)=0$ it follows that

$$
\text { 1) } \sin 2 \pi q=0 ; \quad \text { 2) } \quad J_{1}+4 J_{2} \cos 2 \pi q=0 \text {. }
$$

To be definite we assume $J_{1}<0, J_{2}>0$. Then in the first case $\Phi^{\prime \prime}(q)<0$, if $J_{2}<-J_{1} / 4$. Since $q=0$, then the branching starts from the ferromagnetic phase for $J_{1}, J_{2}$ satisfying

$$
T_{0}^{\prime}=-2 J_{1}-2 J_{2}-4 J_{0} .
$$

In the second case

$$
\begin{aligned}
\cos 2 \pi q_{0} & =-J_{1} / 4 J_{2}, \quad\left|J_{1} / 4 J_{2}\right|<1, \\
\Phi^{\prime \prime}\left(q_{0}\right) & =8 J_{2}\left(J_{1}^{2} / 16 J_{2}^{2}-1\right)<0, \\
T_{0} & =J_{1}^{2} / 4 J_{2}+2 J_{2}-4 J_{0} .
\end{aligned}
$$

Let us consider the range of parameters for which $T_{0}>$ $T_{0}^{\prime}$. These are $J_{1}$ and $J_{2}$ satisfying the inequality $r^{2}>$ $4(-2 r-4)$ with $r=J_{1} / J_{2}$. The latter inequality holds for all real $r$ except for $r=-4$. But $r<0$ and to satisfy (3.3) we suppose

$$
-J_{2} / J_{1}>0.25
$$

Thus, the modulated structures can occur only when inequality (3.4) holds.

Now we prove the small branching theorems for the solutions to equations (3.1), (3.2) with $h=0$. Let $q$ be irrational, then equations (3.2) transform into

$$
\begin{aligned}
& a_{1}=\left(1-t^{2}\right) t^{-2}\left\langle 2 x^{3} / 3+\phi(x), e_{1}(q)\right\rangle, \\
& b_{1}=\left(1-t^{2}\right) t^{-2}\left\langle 2 x^{3} / 3+\phi(x), e_{2}(q)\right\rangle, \\
& a_{l}=\frac{\left(1-t^{2}\right) \Phi(q)}{\Phi(l q)-\left(1-t^{2}\right) \Phi(q)}\left\langle 2 x^{3} / 3+\phi(x), e_{1}(l q)\right\rangle, \\
& b_{l}=\frac{\left(1-t^{2}\right) \Phi(q)}{\Phi(l q)-\left(1-t^{2}\right) \Phi(q)}\left\langle 2 x^{3} / 3+\phi(x), e_{2}(q)\right\rangle, \\
& a_{0}=\frac{\left(1-t^{2}\right) \Phi(q)}{2\left(\Phi(0)-\left(1-t^{2}\right) \Phi(q)\right)}\left\langle 2 x^{3} / 3+\phi(x), \epsilon\right\rangle
\end{aligned}
$$


with

$$
\begin{gathered}
\phi(x)=2\left(\frac{x^{5}}{5}+\cdots+\frac{x^{2 n+1}}{2 n+1}+\cdots\right), \\
T=T_{0}\left(1-t^{2}\right), \quad T_{0}=\Phi(q) .
\end{gathered}
$$

We assume solutions of equations (3.5) to be expressed as

$$
a_{1}=t \bar{a}_{1}, \quad b_{1}=t \bar{b}_{1}, \quad a_{l}=t^{2} \bar{a}_{l}, \quad b_{l}=t^{2} \bar{b}_{l}, \quad a_{0}=t^{2} \bar{a}_{0} .
$$

Then the equations for configurations minimizing free energy become

$$
\begin{aligned}
& \bar{a}_{1}=F_{1}^{(1)}(w)=\frac{1-t^{2}}{2 \pi t^{3}} \int_{0}^{2 \pi} \varphi_{1}(\cdot) \cos \phi d \phi, \\
& \bar{b}_{1}=F_{1}^{(2)}(w)=\frac{1-t^{2}}{2 \pi t^{3}} \int_{0}^{2 \pi} \varphi_{1}(\cdot) \sin \phi d \phi,
\end{aligned}
$$

with

$$
\begin{aligned}
& \bar{a}_{l}=F_{l}^{(1)}(w)=\frac{A_{l}}{2 \pi t^{2}} \int_{0}^{2 \pi} \varphi_{1}(\cdot) \cos \phi d \phi, \\
& \bar{b}_{l}=F_{l}^{(2)}(w)=\frac{A_{l}}{2 \pi t^{2}} \int_{0}^{2 \pi} \varphi_{1}(\cdot) \sin \phi d \phi,
\end{aligned}
$$

$$
\bar{a}_{0}=F_{0}^{(0)}(w)=\frac{A_{0}}{2 \pi t^{2}} \int_{0}^{2 \pi} \varphi_{1}(\cdot) d \phi
$$

$$
w=\left\{t, z, \bar{a}_{1}, \bar{b}_{1}\right\}, z=\{\bar{a}, \bar{b}\}=\left(\left\{\bar{a}_{2}, \bar{b}_{2}\right\}, \cdots,\left\{\bar{a}_{N}, \bar{b}_{N}\right\}, \bar{a}_{0}\right),
$$

$$
\begin{gathered}
A_{l}=\frac{\left(1-t^{2}\right) \Phi(q)}{\Phi(l q)-\left(1-t^{2}\right) \Phi(q)}, l \geq 2, \\
A_{0}=\frac{\left(1-t^{2}\right) \Phi(q)}{2\left(\Phi(0)-\left(1-t^{2}\right) \Phi(q)\right)},
\end{gathered}
$$

$$
\varphi_{1}(\cdot)=\varphi_{1}\left(t^{2} \sum_{l=2}^{N}\left(\bar{a}_{l} \cos l \phi+\bar{b}_{l} \sin l \phi\right)+t^{2} \bar{a}_{0}+t\left(\bar{a}_{1} \cos \phi+\bar{b}_{1} \sin \phi\right)\right) .
$$

To prove small branching theorem for nonlinear equations (3.5), let us proceed by steps. At first we prove the existence of a unique nontrivial solution to system (3.7). Let us introduce the norm

$$
|z|=\max _{2 \leq l \leq N}\left(\left\{\left|\bar{a}_{l}\right|,\left|\bar{b}_{l}\right|\right\},\left|\bar{a}_{0}\right|\right)
$$

and the radius $A$ ball centered at the point

$$
z_{0}=\left(\left\{\bar{a}_{2}^{0}, \bar{b}_{2}^{0}\right\}, \cdots,\left\{\bar{a}_{N}^{0}, \bar{b}_{N}^{0}\right\}, \bar{a}_{0}^{0}\right)
$$

in the $2 N-1$-dimensional space of vectors $\mathrm{z}$, where

$$
\begin{aligned}
& \bar{a}_{l}^{0}=\frac{A_{l}}{2 \pi t^{2}} \int_{0}^{2 \pi} \varphi_{1}\left(t\left(\bar{a}_{1} \cos \phi+\bar{b}_{1} \sin \phi\right)\right) \cos \phi d \phi, \\
& \bar{b}_{l}^{0}=\frac{A_{l}}{2 \pi t^{2}} \int_{0}^{2 \pi} \varphi_{1}\left(t\left(\bar{a}_{1} \cos \phi+\bar{b}_{1} \sin \phi\right)\right) \sin \phi d \phi,
\end{aligned}
$$

$$
\bar{a}_{0}^{0}=\frac{A_{l}}{2 \pi t^{2}} \int_{0}^{2 \pi} \varphi_{1}\left(t\left(\bar{a}_{1} \cos \phi+\bar{b}_{1} \sin \phi\right)\right) d \phi .
$$

Let $|\{\bar{a}, \bar{b}\}| \leq A$ and $\max \left\{\left|\bar{a}_{1}\right|,\left|\bar{b}_{1}\right|\right\} \leq A$ with $A$ be determined later. In operator form (3.7) is

$$
z=F(w),
$$

with the nonlinear operator $F$ given by the r.h.s. of (3.7).

Theorem 1. If $\max \left\{\left|\bar{a}_{1}\right|,\left|\bar{b}_{1}\right|\right\}<A$, then there exists $\alpha>0$ such that $0<t<\alpha$ and the system (3.8) is uniquely solvable by continuously differentiable function of $t, \bar{a}_{1}, \bar{b}_{1}$ in their ranges of values.

For the proof see [10]. The similar theorem is valid for rational $q$.

Theorem 2. For all irrational $q$ that guarantee the validity of

$$
\min _{l}|\Phi(l q)-\Phi(q)|=\delta>0
$$


there is $\alpha_{1}>0$ such that a nonzero solution to system (3.5) exists for $0 \leq t<\alpha_{1}$ if $h=0$. The action of group $G$ (2.13) on this solution gives different solutions of the system (3.5) that are continuously differentiable functions of $t$.

For the proof see [10].

Theorem 3. For all rational $q$ there is $\alpha_{1}^{\prime}>0$ such that there exists nonzero solution of system (3.1) for $0 \leq t<\alpha_{1}^{\prime}$ if $h=0$. The action of group (2.11) on this solution gives different solutions to equations (3.1) that are continuously differentiable functions of $t$.

For the proof see [11].

\section{NEAR CRITICAL MODULATED STRUCTURES $(H \neq 0)$}

Here we consider the case $h \neq 0$ and display the phase transition from the paramagnetic state to the spatially inhomogeneous commensurate configuration or the incommensurate one below the critical magnetic field. In the external magnetic field systems (3.1) and (3.2) have a nonzero solution in the set of vectors $\left\{\left(a_{1}, b_{1}\right), \cdots,\left(a_{N}, b_{N}\right), a_{0}\right\}$ such that

$$
\sum_{l=1}^{N}\left(\left|a_{l}\right|+\left|b_{l}\right|\right)+\left|a_{0}\right|<1
$$

The solution has the form $\left\{(0,0), \cdots,(0,0), \bar{a}_{0}\right\}$ with $\bar{a}_{0}$ solving the equation

$$
-\bar{a}_{0} \Phi(0)+T g^{\prime}\left(\bar{a}_{0}\right) / 2+h=0 .
$$

We assume that

$$
x=\bar{x}+\bar{a}_{0} \epsilon, \quad \bar{x}=\sum_{l=1}^{N}\left(a_{l} e_{1}(l q)+b_{l} e_{2}(l q)\right)+a_{0}^{\prime} \epsilon
$$

$$
g^{\prime}(x)=\sum_{k=1}^{+\infty} \frac{g^{(k)}\left(\bar{a}_{0}\right)}{(k-1) !} \bar{x}^{k-1}=g^{\prime}\left(\bar{a}_{0}\right) \epsilon+g^{\prime \prime}\left(\bar{a}_{0}\right) \bar{x}+\varphi_{2}(\bar{x})
$$

$$
g^{\prime}\left(\bar{a}_{0} \epsilon\right)=g^{\prime}\left(\bar{a}_{0}\right) \epsilon, \quad \bar{x} \epsilon=\bar{x}, \quad \varphi_{2}(\bar{x})=\sum_{k=3}^{+\infty} \frac{g^{(k)}\left(\bar{a}_{0}\right)}{(k-1) !} \bar{x}^{k-1}
$$

Then system (3.1) becomes

$$
\begin{aligned}
& a_{l}\left(T /\left(1-\bar{a}_{0}^{2}\right)-\Phi(l q)\right)\left(1+\delta_{l,\left[\frac{N_{1}+1}{2}\right]}\right)+T\left\langle\epsilon, \varphi_{2}(\bar{x}) e_{1}(l q)\right\rangle=0, \\
& b_{l}\left(T /\left(1-\bar{a}_{0}^{2}\right)-\Phi(l q)\right)\left(1-\delta_{l,\left[\frac{N_{1}+1}{2}\right]}\right)+T\left\langle\epsilon, \varphi_{2}(\bar{x}) e_{2}(l q)\right\rangle=0, \\
& 2 a_{0}^{\prime}\left(T /\left(1-\bar{a}_{0}^{2}\right)-\Phi(0)\right)+T\left\langle\epsilon, \varphi_{2}(\bar{x})\right\rangle=0, \quad l=\overline{1, N},
\end{aligned}
$$

and system (3.2) becomes

$$
\begin{aligned}
& a_{l}\left(T /\left(1-\bar{a}_{0}^{2}\right)-\Phi(l q)\right)+T\left\langle\epsilon, \varphi_{2}(\bar{x}) e_{1}(l q)\right\rangle=0, \\
& b_{l}\left(T /\left(1-\bar{a}_{0}^{2}\right)-\Phi(l q)\right)+T\left\langle\epsilon, \varphi_{2}(\bar{x}) e_{2}(l q)\right\rangle=0, \\
& 2 a_{0}^{\prime}\left(T /\left(1-\bar{a}_{0}^{2}\right)-\Phi(0)\right)+T\left\langle\epsilon, \varphi_{2}(\bar{x})\right\rangle=0 .
\end{aligned}
$$

The spatially inhomogeneous solution to systems (3.1), (3.2) exists if systems (4.2), (4.3) have a nonzero solution. The first degeneration of the spectrum of the linearized part of the nonlinear operator (4.2) or (4.3) occurs when

$$
\begin{aligned}
T /\left(1-\bar{a}_{0}^{2}\right)-\Phi(q) & =0, \\
-\bar{a}_{0} \Phi(0)+T g^{\prime}\left(\bar{a}_{0}\right) / 2+h & =0 .
\end{aligned}
$$

If there exists a solution to system (4.4), then zero solu- tion for systems (4.2), (4.3) bifurcates. It means a transition from the paramagnetic phase to the modulated structure. As a result system (4.4) gives single equivalent equation

$T=\Phi(q)-$

$$
\frac{\Phi(q) h^{2}}{\left(\Phi(0)-\frac{1}{2} T(1-T / \Phi(q))^{-\frac{1}{2}} g^{\prime}(\sqrt{1-T / \Phi(q)})\right)^{2}}
$$

From (4.5) it follows Lemma 4. The critical magnetic field for phase transition from the paramagnetic state to the modulated structure is equal to $|\Phi(0)|$.

For the proof see [12].

Having in mind that mean-field theory critical exponent $\beta$ equals $1 / 2$ we suppose the deviation from the 
critical temperature to be standard. Therefore

$$
\lambda^{2}(T, h)=\Phi(q)-T /\left(1-\bar{a}_{0}^{2}\right)
$$

where $\bar{a}_{0}$ solves equation (4.1). If $T_{0}(h)$ solves equation (4.5), then $\lambda^{2}\left(T_{0}(h), h\right)=0$.

In terms of the new variables system (4.3) for the irrational $q$ takes the form of

$$
\begin{aligned}
& -\lambda^{2} a_{1}+T\left\langle\epsilon, \varphi_{2}(\bar{x}) e_{1}(q)\right\rangle=0, \\
& -\lambda^{2} b_{1}+T\left\langle\epsilon, \varphi_{2}(\bar{x}) e_{2}(q)\right\rangle=0,
\end{aligned}
$$

$$
\begin{aligned}
& a_{l}\left(\Phi(q)-\Phi(l q)-\lambda^{2}\right)+T\left\langle\epsilon, \varphi_{2}(\bar{x}) e_{1}(l q)\right\rangle=0 \\
& b_{l}\left(\Phi(q)-\Phi(l q)-\lambda^{2}\right)+T\left\langle\epsilon, \varphi_{2}(\bar{x}) e_{2}(l q)\right\rangle=0 \\
& 2 a_{0}^{\prime}\left(\Phi(q)-\Phi(0)-\lambda^{2}\right)+T\left\langle\epsilon, \varphi_{2}(\bar{x})\right\rangle=0
\end{aligned}
$$

We seek the solution of systems (4.6), (4.7) in the form of

$$
a_{1}=\lambda \tilde{a}_{1}, \quad b_{1}=\lambda \tilde{b}_{1}, \quad a_{l}=\lambda^{2} \tilde{a}_{l}, \quad b_{l}=\lambda^{2} \tilde{b}_{l}, \quad a_{0}^{\prime}=\lambda^{2} \tilde{a}_{0}^{\prime} .
$$

Then we rewrite $(4.6),(4.7)$ as

$$
\begin{gathered}
\tilde{a}_{1}=F_{1}^{(1)}(\tilde{w})=\frac{T}{\lambda^{3}} \sum_{k=3}^{+\infty} \frac{g^{(k)}\left(\bar{a}_{0}\right)}{(k-1) !} \lambda^{k-1}\left\langle\epsilon,(\cdot)^{k-1} e_{1}(q)\right\rangle \\
\tilde{b}_{1}=F_{1}^{(2)}(\tilde{w})=\frac{T}{\lambda^{3}} \sum_{k=3}^{+\infty} \frac{g^{(k)}\left(\bar{a}_{0}\right)}{(k-1) !} \lambda^{k-1}\left\langle\epsilon,(\cdot)^{k-1} e_{2}(q)\right\rangle \\
\tilde{a}_{l}=F_{l}^{(1)}(\tilde{w})=\frac{T}{\lambda^{2}\left(\lambda^{2}+\Phi(l q)-\Phi(q)\right)} \sum_{k=3}^{+\infty} \frac{g^{(k)}\left(\bar{a}_{0}\right)}{(k-1) !} \lambda^{k-1}\left\langle\epsilon,(\cdot)^{k-1} e_{1}(l q)\right\rangle, \\
\tilde{b}_{l}=F_{l}^{(2)}(\tilde{w})=\frac{T}{\lambda^{2}\left(\lambda^{2}+\Phi(l q)-\Phi(q)\right)} \sum_{k=3}^{+\infty} \frac{g^{(k)}\left(\bar{a}_{0}\right)}{(k-1) !} \lambda^{k-1}\left\langle\epsilon,(\cdot)^{k-1} e_{2}(l q)\right\rangle, \\
\tilde{a}_{0}^{\prime}=F_{0}^{(0)}(\tilde{w})=\frac{T}{2 \lambda^{2}\left(\lambda^{2}+\Phi(0)-\Phi(q)\right)} \sum_{k=3}^{+\infty} \frac{g^{(k)}\left(\bar{a}_{0}\right)}{(k-1) !} \lambda^{k-1}\left\langle\epsilon,(\cdot)^{k-1}\right\rangle,
\end{gathered}
$$

where

$$
\begin{aligned}
& \tilde{w}=\left\{\lambda, \tilde{z}, \tilde{a}_{1}, \tilde{b}_{1}\right\}, \quad \tilde{z}=\{\tilde{a}, \tilde{b}\}=\left(\left\{\tilde{a}_{2}, \tilde{b}_{2}\right\}, \cdots,\left\{\tilde{a}_{N}, \tilde{b}_{N}\right\}, \tilde{a}_{0}^{\prime}\right), \\
& (\cdot)=\left(\lambda \sum_{l=2}^{N}\left(\tilde{a}_{l} e_{1}(l q)+\tilde{b}_{l} e_{2}(l q)\right)+\lambda \tilde{a}_{0}^{\prime} \epsilon+\tilde{a}_{1} e_{1}(q)+\tilde{b}_{1} e_{2}(q)\right) .
\end{aligned}
$$

Let us consider the norm

$$
|\tilde{z}|=\max _{2 \leq l \leq N}\left\{\left|\tilde{a}_{l}\right|,\left|\tilde{b}_{l}\right|,\left|\tilde{a}_{0}^{\prime}\right|\right\}
$$

and the radius $A$ ball centered at the point

$$
\tilde{z}_{0}=\left(\left\{\tilde{a}_{2}^{0}, \tilde{b}_{2}^{0}\right\}, \cdots,\left\{\tilde{a}_{N}^{0}, \tilde{b}_{N}^{0}\right\}, \tilde{a}_{0}^{0}\right)
$$

in the set of vectors $\tilde{z}$, where

$$
\tilde{a}_{l}^{0}=\frac{T}{\lambda^{2}+\Phi(l q)-\Phi(q)} \sum_{k=3}^{+\infty} \frac{g^{(k)}\left(\bar{a}_{0}\right)}{(k-1) !} \lambda^{k-3}\left\langle\epsilon,(\cdots)^{k-1} e_{1}(l q)\right\rangle
$$




$$
\begin{aligned}
& \tilde{b}_{l}^{0}=\frac{T}{\lambda^{2}+\Phi(l q)-\Phi(q)} \sum_{k=3}^{+\infty} \frac{g^{(k)}\left(\bar{a}_{0}\right)}{(k-1) !} \lambda^{k-3}\left\langle\epsilon,(\cdots)^{k-1} e_{2}(l q)\right\rangle, \\
& \tilde{a}_{0}^{\prime 0}=\frac{T}{2\left(\lambda^{2}+\Phi(0)-\Phi(q)\right)} \sum_{k=3}^{+\infty} \frac{g^{(k)}\left(\bar{a}_{0}\right)}{(k-1) !} \lambda^{k-3}\left\langle\epsilon,(\cdots)^{k-1}\right\rangle,
\end{aligned}
$$

$$
(\cdots)=\left(\tilde{a}_{1} e_{1}(q)+\tilde{b}_{1} e_{2}(q)\right)
$$

Let $F(\tilde{w})$ be a nonlinear operator given by the right part of system (4.9), then this system turns to be

$$
\tilde{z}=F(\tilde{w})
$$

\section{Theorem 4. Let}

$\min _{l}|\Phi(l q)-\Phi(q)|=\delta>0, \quad l=0,2,3,4, \cdots, N-1, N$,

and $\lambda_{0}>0$ satisfies the inequality

$$
\begin{aligned}
& \max _{l} \frac{T}{\left|\lambda^{2}+\Phi(l q)-\Phi(q)\right|}(2 N-1) \lambda \times \\
& \times \sum_{k=3}^{+\infty} \frac{\left|g^{(k)}\left(\bar{a}_{0}\right)\right|}{(k-2) !} \lambda^{k-3}((2 N-1) A \lambda+2 A)^{k-2}<1,
\end{aligned}
$$

then for all $\lambda \in\left[0, \lambda_{0}\right]$ and $\tilde{a}_{1}, \tilde{b}_{1}, h$ such that

$$
\tilde{a}_{1}^{2}+\tilde{b}_{1}^{2} \leq A^{2}, \quad|h|<|\Phi(0)|
$$

there exist the critical temperature $T_{0}(h)$ and an interval $\left(T_{0}(h)-\lambda^{2}, T_{0}(h)\right)$ on which the solution of nonlinear equations (4.10) exists being a continuously differentiable function of $\lambda \in\left[0, \lambda_{0}\right]$ and $\tilde{a}_{1}, \tilde{b}_{1}$ for $\tilde{a}_{1}^{2}+\tilde{b}_{1}^{2} \leq A^{2}$.

For the proof see [12].A similar theorem is valid for the rational $q$.

Remark. Continuous differentiability with respect to the variables $\lambda$ and $\tilde{a}_{1}, \tilde{b}_{1}$ results from continuous differentiability of the successive approximations and their uniform convergence to the solution.

Theorem 5. For all irrational $q$ that guarantee the validity of

$$
\min _{l}|\Phi(l q)-\Phi(q)|=\delta>0
$$

there is such $\lambda_{1}>0$, that system (4.3) is solvable for

$$
0<\lambda<\lambda_{1}, \quad|h|<|\Phi(0)| .
$$

The action of group $G(2.13)$ on this solution gives different solutions of system (4.3) being continuously differ- entiable functions of $\lambda$.

For the proof see [12].

Theorem 6. For those rational $q$, for which $N_{1} \neq 3$, there is $\lambda_{0}>0$ such that a nonzero solution to system (4.2) exists for

$$
0 \leq \lambda<\lambda_{0}, \quad|h|<|\Phi(0)|
$$

The action of group (2.11) on this solution gives different solutions to system (4.2) being continuously differentiable functions of $\lambda$.

For the proof see [13].

\section{GLOBAL INCOMMENSURATE EXTREMUM}

For $N=1$ conditions of Theorems 4 and 5 hold. In this case one can construct not only a solution near the critical temperature but also the global solution. For $N=1$ configuration (2.1) takes the form of

$$
x=a_{1} e_{1}(q)+b_{1} e_{2}(q)+a_{0} \epsilon .
$$

The specific free energy for this configuration is

$$
\begin{aligned}
F_{h} & =-\frac{1}{2}\left(\frac{1}{2}\left(a_{1}^{2}+b_{1}^{2}\right) \Phi(q)+a_{0}^{2} \Phi(0)\right) \\
& +h a_{0}+\frac{1}{2} T\langle\epsilon, g(x)\rangle .
\end{aligned}
$$

Having calculated the average

$$
\langle\epsilon, g(x)\rangle=\frac{1}{2 \pi} \int_{0}^{2 \pi} g\left(u \cos \varphi+a_{0}\right) d \varphi
$$

with $u=\left(a_{1}^{2}+b_{1}^{2}\right)^{\frac{1}{2}}$ we have [14]

$$
\begin{aligned}
& F_{h}=-\frac{1}{2}\left(\frac{1}{2} u^{2} \Phi(q)+a_{0}^{2} \Phi(0)\right)+h a_{0} \\
& +\frac{T}{2}\left(2-\sqrt{\left(1+a_{0}\right)^{2}-u^{2}}-\sqrt{\left(1-a_{0}\right)^{2}-u^{2}}-2 \ln 2\right. \\
& +\left(1+a_{0}\right) \ln \left(1+a_{0}+\sqrt{\left(1+a_{0}\right)^{2}-u^{2}}\right)
\end{aligned}
$$




$$
\left.+\left(1-a_{0}\right) \ln \left(1-a_{0}+\sqrt{\left(1-a_{0}\right)^{2}-u^{2}}\right)\right) .
$$

For $h=0$ the free energy is

$$
\begin{aligned}
& F=-\frac{1}{4} u^{2} \Phi(q)+T\left(1-\sqrt{1-u^{2}}+\right. \\
& \left.\ln \left(1+\sqrt{1-u^{2}}\right)-\ln 2\right) .
\end{aligned}
$$

The equation for the configuration minimizing the free energy (5.4) is

$$
-\frac{1}{2} \Phi(q)+\frac{T}{1+\sqrt{1-u^{2}}}=0
$$

From here we obtain the global solution

$$
u^{2}=1-(2 T / \Phi(q)-1)^{2},
$$

terminating at $u^{2}=1$, i.e. the termination temperature is equal to $\Phi(q) / 2$. Taking into account (5.6) we write the free energy of the incommensurate phase (5.4) as

$$
F=T(1-T / \Phi(q)+\ln (T / \Phi(q))) .
$$

The configuration minimizing the free energy (5.3) satisfies equations

$$
\begin{aligned}
& \Phi(q)+T\left(\frac{1}{1+a_{0}+\sqrt{\left(1+a_{0}\right)^{2}-u^{2}}}+\frac{1}{1-a_{0}+\sqrt{\left(1-a_{0}\right)^{2}-u^{2}}}\right)=0, \\
& -a(0)+h+\frac{T}{2} \ln \frac{1+a_{0}+\sqrt{\left(1+a_{0}\right)^{2}-u^{2}}}{1-a_{0}+\sqrt{\left(1-a_{0}\right)^{2}-u^{2}}}=0
\end{aligned}
$$

or

$$
\begin{aligned}
a_{0} & =\frac{T}{\Phi(q)} \sinh \left(2 T^{-1}\left(a_{0} \Phi(0)-h\right)\right)-\tanh \left(T^{-1}\left(a_{0} \Phi(0)-h\right)\right) \\
u^{2} & =\left(2\left(1+a_{0}\right)-\frac{T}{\Phi(q)}\left(1+\exp \left(2 T^{-1}\left(a_{0} \Phi(0)-h\right)\right)\right)\right) \\
& \times \frac{T}{\Phi(q)}\left(1+\exp \left(2 T^{-1}\left(a_{0} \Phi(0)-h\right)\right)\right) .
\end{aligned}
$$

Theorem 7. There exists the global solution minimizing free energy (5.3) for a rather small $h$.

For the proof see [14].

Theorem 8. The global solution minimizing free energy (5.3) terminates when the amplitude of incommensurate configuration is

$$
u=\left(a_{1}^{2}+b_{1}^{2}\right)^{\frac{1}{2}}=1-\left|\hat{a}_{0}\right|
$$

where $\hat{a}_{0}$ solves the equation

$$
\frac{\Phi(q)}{4} \ln \frac{1+\sqrt{\left|a_{0}\right|}}{1-\sqrt{\left|a_{0}\right|}}\left(1-\left|a_{0}\right|\right)-\frac{\Phi(0)\left|a_{0}\right|+|h|}{1+\sqrt{\left|a_{0}\right|}}=0
$$

For the proof see [14].

Theorem 9. There exists phase transition of the first kind from the incommensurate phase into the ferromagnetic one. For $h \neq 0$ the existence condition of phase transition is

$$
\begin{aligned}
\Phi(q) & <\left(1-\hat{a}_{0}-\left(1+\sqrt{\hat{a}_{0}}\right)\left(2\left(1+\left(1+\hat{a}_{0}\right) \ln \left(1+\sqrt{\hat{a}_{0}}\right)-\sqrt{\hat{a}_{0}}-\ln 2\right)\right.\right. \\
& \left.\left.+\left(1-\hat{a}_{0}\right) \ln \left(1-\hat{a}_{0}\right)\right)\right)^{-1} 2\left(\Phi(0)\left(1+\hat{a}_{0}\right)-2 \hat{h}\right),
\end{aligned}
$$


where $\hat{a}_{0}$ and $\hat{h}$ solve equation (5.10). For $h=0$ this condition has the form of

$$
\Phi(q)<\Phi(0) /(\ln 2-1 / 2)
$$

For the proof see [14].

[1] W. Selke, Phys. Rep. 170, 213 (1988).

[2] M. Høgh Jensen, P. Bak, Phys. Rev. B 27, 6853 (1983).

[3] K. Nakanishi, J. Phys. Soc. Jpn. 58, 1296 (1989).

[4] N. S. Gonchar, W. H. Kozyrski, Ukr. Math. J. 43, 1509 (1991).

[5] C. S. O. Yokoi, Phys. Rev. B 43, 8487 (1991).

[6] N. S. Gonchar, W. H. Kozyrski, Ukr. Phys. J. 36, 1857 (1991).

[7] N. S. Gonchar, W. H. Kozyrski, Ukr. Phys. J. 37, 449 (1992).

[8] J. Villain, J. Phys. Chem. Solids 11, 303 (1959).
[9] N. S. Gonchar, W. H. Kozyrski, Preprint ITP-87-5R, Kiev, 1987.

[10] N. S. Gonchar, W. H. Kozyrski, Preprint ITP-90-51R, Kiev, 1990.

[11] N. S. Gonchar, H. G. Hajduk, W. H. Kozyrski, Preprint ITP-95-2U, Kiev, 1995.

[12] N. S. Gonchar, H. G. Hajduk, Preprint ITP-94-31U, Kiev, 1994.

[13] H. G. Hajduk, Preprint ITP-95-22U, Kiev, 1995.

[14] H. G. Hajduk, Preprint ITP-95-5U, Kiev, 1995.

\title{
ВИЧЕРПНИЙ АНАЛІЗ МАГНЕТНИХ НАДСТРУКТУР В АНІЗОТРОПНИХ СИСТЕМАХ
}

\author{
М. Гончар, В. Козирський, Г. Гайдук \\ Відділ математичного моделювання Інституту теоретичной фізики \\ імені М. М. Боголюбова НАН Украӥни, Украйна, 252143, Київ, вул. Метрологічна, 14 б
}

\begin{abstract}
Розвинуто новий метод аналітичного розгляду модульованих надструктур у модельних магнетних системах, близьких до критичності. Встановлено умови для переходу парамагнет-(не)співмірна фаза у класі анізотропних моделей Ізінга з зовнішнім магнетним полем та без нього. Знайдено його критичного значення. Виведено нове представлення питомої вільної енергії для співвимірних та неспіввимірних конфігурацій та виявлено іiі приховану симетрію. Доведено існування глобального розв'язку для неспіввимірної конфігурації, що мінімізує вільну енергію. Встановлені умови зникнення глобального розв'язку. Доведено, що існування фазового переходу від неспіввимірної до феромагнетної фази залежить від обмінних інтегралів.
\end{abstract}

